Review

\title{
Management of infertility: an overview
}

\author{
Farouk Mahmoud ${ }^{1}$ \\ Sri Lanka Journal of Obstetrics and Gynaecology 2012; 34: 168-173
}

\section{Introduction}

Healthy sperms, eggs, fallopian tubes, uterine milieu and receptive endometrium constitute the essentials for conception. Failure in one or more of these leads to infertility which is defined as failure to achieve a pregnancy within 12 months of regular coitus. The incidence of infertility is around $8-10 \%$ (Lass 1999). Semen abnormalities (35\%), tubal and pelvic pathology $(35 \%)$, ovulatory $(15 \%)$ and unexplained infertility $(10 \%)$ dominate the list of causes of infertility.

\section{Management of infertility}

Systemic disease should be excluded in both partners and it is important to commence investigations and treatment early because of declining ovulation with age.

Evaluation of male infertility: Relevant questions are a history of mumps, torsion or haematoma of testis, inguinal or scrotal surgery and gonadotoxins such as chemotherapy, radiation and exogenous androgenic steroids. Physical examination comprises size, consistency and undescended testes, absence of vas and varicocele.

Semen analyses (SA) is performed after 3 days abstinence and examined within 2 hours. Normal parameters constitute a volume of between 2.0 and $5.0 \mathrm{ml}$., concentration of $>20$ million per ml., motility of $50 \%$ and normal morphology $>20 \%$. White blood cells or bacteria indicate infection and require culture.

If SA is abnormal, serum assay for FSH and LH (necessary to produce sperm and testosterone respectively), testosterone and prolactin are performed along with chromosome analysis in severe oligo- or azoospermia.

The commonest cause of male infertility is primary testicular failure with small testes and high $\mathrm{FSH}$, the aetiology of which remains unclear.

\footnotetext{
${ }^{1}$ Consultant Gynaecologist and IVF Specialist, Durdans Hospital and Nawaloka Hospital, Colombo, Sri Lanka.
}

Secondary causes are related to pituitary deficiencies, thermal injury (cryptorchidism), chemical (occupation), hypoxia (torsion and haematoma), absence of vas deferens, viral (mumps), genetic factors and autoimmunity. Unlike primary testicular failure, pituitary defect is amenable to replacement therapy, cryptorchidism by early treatment, chemical toxins by avoidance of exposure and hypoxia by prompt treatment of torsion and haematoma but currently the last three may require special sperm retrieval techniques. With counts of $>1$ million and reasonable motility and morphology, IVF with ICSI (intra-cellular sperm injection) should give good results.

\section{Female infertility}

In the history it is important to inquire whether infertility is primary or secondary, age, duration of infertility, coital frequency and regularity of menstrual cycles. Important features in examination are BMI, hirsutism, uterine size and pelvic mass.

The causes of female infertility are abnormalities in ovulation, patency and function of fallopian tubes and endometrial receptivity.

Investigations comprise early follicular phase blood assay for FSH, LH, ultra-sound of pelvis (preferably transvaginal), hysteroscopy and laparoscopy if necessary.

Ovulation problems could be due to polycystic ovarian disease (PCOD) which will need weight reduction if $\mathrm{BMI}$ is $>30$ and clomiphene to induce ovulation. Endo-crinological (hypothalamic/pituitary dysfunction, hypo or hyperthyroidism and hyperprolactinaemia) causes which will require appropriate treatment while the only option for premature ovarian failure and chromosomal oocyte dysfunction is ovum or embryo donation.

Intra-uterine adhesions (Asherman's syndrome), uterine septa and submucus fibroids require hysteroscopic excision.

Hydrosalpinges and ART: The 'flushing' effect and embryo-toxins of fluid released from hydrosalpinges may hinder implantation. Management is by laparoscopic excision or occlusion of the tubes. 
In cases of tubal damage or obstruction, microsurgical repair with reconstruction and refashioning of damaged fallopian tubes at laparoscopy or laparotomy should be attempted in suitable cases.

Receptivity of endometrium: In practice endometrial thickness with the triple sign is taken as a reflection of endometrial receptivity. Although this indicates a suitable time for transfer of the embryo, it does not say anything about the architecture, chemical, endocrine or immunological status necessary for implantation.

Unexplained infertility - The diagnosis of unexplained infertility is made by exclusion where there is no discernible abnormality in both male and female (Some cases of unexplained infertility may be due to sperm-zona dysfunction responsible for fertilisation failure - Schatten 1999). Management is by follicular tracking by trans-vaginal ultrasound to synchronise coitus with natural or induced ovulation so as to enhance fertilisation. If no response after 3-6 months or earlier if the age of female is $>35$ years, proceed to IUI-H.

Intra-uterine insemination - husband (IUI-H) bypasses the sometimes 'noxious' effect of sperm antibodies in the cervical mucus and facilitates the passage of sperm into the fallopian tube. This is continued for 6 months or shorter if the age of female is >35 years. DI (donor insemination) for IUI is discussed below.

In the final analysis, while many were treated success-fully, those with significant ovarian, tubal or sperm dysfunction, including prolonged and unexplained infertility had little hope of fecundity and will need IVF. IVF is not entirely complication-free. Moreover, IVF does not cure the infertility; it temporarily bridges the infertility gap. It is important to make the couple aware of these and frequent visits, multiple injections and most important the cost of IVF.

\section{In vitro fertilisation (IVF)}

The main indications for IVF are severely damaged fallopian tubes, oligo-astheno-terato-zoospermia) and unexplained infertility.

Response to stimulation: Prior to embarking on IVF it is prudent to assess how the ovaries are going to respond. AMH (Anti-Mullerian Hormone) is an excellent but expensive tool while previous response, age, FSH, antral follicular count (AFC) and ovarian volume have reasonable predictive value.

\section{IVF protocol}

Currently, IVF technique involves initial downregu-lation of the pituitary with gonadotrophic releasing hormone (GnRh) agonists or antagonists. Once the pituitary is suppressed the ovaries are stimulated with follicle stimulating hormone (FSH) to produce multiple follicles. Follicular response is monitored by ultra-sound and oestrogen assay. An artificial luteinising hormone (LH) surge is triggered by the administration of human chorionic gonadotropin (HCG) and 36 hours later the eggs are harvested by transvaginal, ultrasound-directed aspiration. The eggs are fertilised in vitro (IVF) with or without intracytoplasmic sperm injection (ICSI), followed by embryo transfer at the pro-nucleate stage on day 2-3 or blastocyst stage on day 5 or 6 . Induction protocols using GnRH analogues often led to a luteal phase defect and required progesterone support administered, usually as vaginal suppo-sitories, to support the pregnancy till 12-16 weeks.

\section{Pituitary suppression}

Oral contraceptives enable the timing of IVF cycle to be regulated. Pre-treatment with GnRH agonists prevents premature surge of $\mathrm{LH}$ which results in premature ovulation and cancellation of the cycle. However, the initial 'flare' effect of GnRH agonists is not desirable in most women. GnRH antagonists, a later innovation in the genre of pituitary suppressants, avoid a flare-effect by immediate down regulation and also minimise the risk of OHSS. Both GnRH agonists and antagonists provide better control and smoother and more reliable follicle stimulation with more eggs and more importantly more pregnancies (Tarlatzis and Bili 2001). GnRH antagonists prevent ovarian cyst formation with less need for luteal support and with less medication but the pregnancy rates are similar for both (Tarlatzis and Kolibianakis 2007).

\section{Ovulation induction}

Ovarian hyper-stimulation syndrome (OHSS) and multiple pregnancies are avoided by tailoring the dose of FSH according to age, PCO (polycystic ovaries), serum AMH and FSH level, past history, ovarian volume, antral follicle count and body mass index (BMI).

\section{Triggering an artificial LH surge}

An LH surge is triggered with 5-10,000 units of HCG (human chorionic gonadotrophin). Recent studies indicate that in cycles suppressed with GnRh anta-gonist triggering by $\mathrm{GnRh}$ agonist is effective and minimises OHSS and luteal phase defect. 


\section{Ovum pickup}

Compared to the more invasive, less accurate and cumbersome laparoscopic aspiration of eggs, transvaginal ultrasound-guided aspiration of follicles provides easier, accurate and more efficient harvesting of eggs (Wickland and Hamberger 1985).

\section{Sperms}

The husband's sperms are obtained on the day of ovum pickup. Frozen specimens are thawed where the husband cannot produce a sample on demand or be present, where sperms were procured by special sperm retrieval techniques or stored pre-chemo or radiotherapy.

\section{Fertilisation}

In cases of severe oligo-astheno-terato-zoospermia spontaneous IVF is poor. Early attempts to facilitate fertilisation with partial zona dissection (PZD) and sub-zonal injection (SUZI).

Were only marginally successful. The technique of intra-cytoplasmic sperm injection (ICSI) involves injecting a single sperm, even a non-motile one, directly into the ooplasm (Palermo, Joris, Devroey and Van Steirteghem 1992). ICSI has revolutionised fertilization and improved fertilisation rates resulting in more good quality embryos (Palermo et al 2001).

In azoospermia, special sperm retrieval techniques of epididymal sperm aspiration (MESA), percutaneous epidymal sperm aspiration (PESA), testicular sperm aspiration (TESA) or extraction (TESE) are employed along with ICSI.

\section{Embryo culture}

Good culture systems incorporating improved incu-bating chambers, environment control and extended culture with sequential appropriatelyconstituted media, tailored for cleavage up to blastocyst-stage has optimised embryo quality (Gardner and Lane 2001).

\section{Embryo transfer}

Successful transfer requires ultrasound guided atrau-matic correct placement of the embryo (Schoolcraft 2001).

Choosing optimal embryos for transfer - quality embryos can be chosen by zygote scoring or blastocyst transfer (Scott and Smith 1998; Gardener et al 1998) and if indicated, pre-implantation genetic diagnosis (PGD). Blastocyst transfer is physiological, screens some chromosomally abnormal and poor quality embryos. This is recommended if there are $>4$ embryos on day 3. With fresh single blastocyst transfer birth rate has remained high, and multiple birth rate has been reduced to $<10 \%$, less than half the Australian national average and with two-thirds of the miscarriage rate (Jansen 2006).

Gamete intra-fallopian transfer (GIFT), where sperm plus eggs and zygote intra-fallopian transfer (ZIFT), where embryos are transferred at laparoscopy into the fallopian tubes, are variations of IVF. Though initially more successful than IVF, the improved technology of IVF now produces equal or better results without resorting to invasive laparoscopy. Hence the pre-ference today of IVF over GIFT and ZIFT.

\section{Implantation}

Apart from transferring quality embryos implantation can be enhanced by improving endometrial milieu. Endometrial receptivity is governed by adequate blood supply, appropriate architecture, nutrients for sustenance of the pre-embryo, immunological and chromosomal factors and complex signals and dialogue between the embryo and endometrium to facilitate apposition, adhesion and invasion, the essential triad for successful implantation. Although endometrial thickness is reassuring, accurate bio-markers for assessment of endometrial receptivity have been elusive to date.

Assisted hatching - Assisted hatching by partial zona dissection enhances the release of the blastocyst from within the zona. It could be performed at the 6-8 cell or blastocyst-stage by chemical or laser technique (Cohen et al 1990). Assisted hatching increases the implantation and pregnancy rates in previous implantation failures but has no place for first IVF attempts, while its role in advanced age, 'thick zona' and frozenthawed embryos remain uncertain (Velga and Boiso 2001).

Immunological influences in implantation-failure are not totally unexpected given the immunologically and genetically unrelated embryo and host endometrial tissue. However, the role of intravenous immuno-globin for repeat implantation failure remains controversial.

Anti-thrombogenic therapy with aspirin and heparin is helpful in abnormal implantation, placentation and early embryonic vascular compromise with (Damario and Rosenwaks 2001).

\section{Luteal support}

With GnRh agonists and HCG protocol, luteal support with progestagen is necessary. However, the use of GnRh antagonists with GnRh agonist as 
triggering agent reduces OHSS and the need for luteal support (Fauser et al 2002).

\section{Cryo-storage of sperms, eggs and embryos}

Vitrification enables eggs and embryos to be frozen with minimal damage.

Freezing has many uses in ART enabling the storage of eggs, embryos, immature oocytes and more recently ovarian tissue whenever pregnancy needs to be deferred for social reasons and prior to chemo or radiotherapy and finally to preserve donor embryos. An additional bonus of saving embryos derived from eggs obtained at an early age is the reduced incidence of aneuploidy.

Clinical indications for storing sperms are problems of masturbation 'on demand', following sperm retrieval for ICSI, prior to chemo- or radiotherapy, pre-vasectomy and for sperm donation.

\section{Complications of IVF}

The main complications are ovarian hyper-stimulation syndrome (OHSS) a potentially life-threatening condition), multiple pregnancies (leading to extreme prematurity with neonatal deaths and prolonged neonatal care), ovarian and vascular trauma, security concerns, carcinogenic effects of ART medications (now disproved) and psychological sequelae for the couple.

\section{Third-party participation in ART Donation of genetic material}

Where one or other (or rarely both) partners lack their own genetic potential, the only current option is donation of sperm, ova or embryo.

Sperm donation - This is indicated no sperm could be obtained even with special soerm retrieval techniques. With the introduction of intra-cytoplasmic sperm injection (ICSI) in 1990), the need for sperm donation has dropped dramatically.

Ovum donation may be needed for women who are older, oligo or anovulatory, premature menopause, fertilisation failure, recurrent miscarriage, postmenopausal or who have genetic defects. Other recipients are women with post radio- or chemo-therapy anovulation or those with no ovaries.

Embryo donation: Indications for this comprise the above coupled with severe male infertility.

Pregnancy in the older female: Fertility is markedly compromised several years before the menopause, suggesting that factors other than complete depletion of the follicular store play a role in the loss of oocyte competency in 'ageing' women (Serhal 2004). The use of higher doses of gonadotrophins coupled with the 'flare-effect' of GnRH agonists and reducing the dose may be helpful. Embryo cryo-preservation while still young provides the most successful option, freezing mature and immature oocytes and ovarian tissue with subsequent in-vitro maturation or autologous transplantation offer additional avenues ( $\mathrm{Ng}$ and $\mathrm{Ho} 2007$ ). The post-menopausal uterus does respond well when supported by steroid therapy and enable embryos to implant efficiently (Antinori et al 1993).

Aneuploidy is present in $50 \%$ of early IVF embryos which increases with maternal age. Pre-implantation genetic diagnosis to exclude aneuploid embryos may also be useful.

However, the logical deduction that aneuploidy screening will increase implantation and pregnancy rates and reduce miscarriages has not yet been proven (Staessen et al 2004). Even though it is early days, rejuvenating the 'ageing' cytoplasm with donor cytoplasm containing mRNA, proteins and mitochondria, one-third of repeat IVF failures, supposedly due to poor embryo development, fell pregnant with this technique (Cohen et al 1997).

Posthumous parenting: The use of stored gametes or embryos after the death of one or other (or rarely both) partners is possible though a concern in ethical and theological circles and a minefield in the court-room.

\section{Surrogacy}

The surrogate may or may not contribute her eggs or the sperm, egg or embryo may be from the commissioning couple or donor. Surrogacy may lead to ethical, religious and legal complications (Rothenberg 1988).

\section{Preservation of reproductive potential}

Indications are to defer pregnancies, pre- chemoor radiotherapy and where premature ovarian failure is anticipated. Eleanora Porcu (2001) quotes a postthaw survival rate of $57 \%$ after cryo-preservation of eggs. In vitro maturation (IVM) of immature oocytes from biopsied or surgically removed ovaries and more recently by transvaginal aspiration of antral follicles is promising (Suikkari and Soderstrom-Antttila 2007). Preservation of male reproductive potential.

Prompt freezing in patients with haematoma and torsion of testes and prior to vasectomy may avoid the need for sperm donation.

\section{Pre implantation genetic diagnosis (PGD)}

In PGD, blastomere biopsy and analysis of single cells by polymerase chain reaction (PCR) and 
fluorescent in-situ hybridisation (FISH) is performed (Lavery and Winston 1999).

Fifty per cent of spontaneous early pregnancy losses show chromosomal abnormalities, and more than $90 \%$ of chromosomally abnormal pregnancies miscarry (Grace, El-Toukhy and Scriven et al 2006). Indications for PGD are older woman, known chromosomal abnormality, recurrent IVF failure and pregnancy loss.

\section{Future technologies in reproductive medicine}

Transplantation of stored ovarian tissue has been achieved (Donnez et al - 2004) and Mierow et al - 2005) while uterine transplantation, gene therapy, stem cell technology (SCT) and therapeutic cloning are all in the research phase but clinical applications are not too far away.

\section{Conclusion}

In the past quarter of a century, exponential advances in reproductive medicine with improvements in ovulation induction, ultrasonography, culture systems, fertilisation and molecular biology have led to safer and more successful methods with expanded scope to investigate and treat many more types of infertility.

Infertility management has improved and now offers a chance for almost all kinds of infertility including those who lack sperm, eggs or uterus, unexplained infertility, recurrent fertilisation-failure or pregnancy loss or have genetically abnormal conceptions.

Future advances in transplantation technology and stem cell technology will offer avenues for all women to 'have their own uterus' and their own genetic make-up for their progeny.

\section{References}

Antinori S, Versaci C, Gholami GH, et al. Oocyte donation in menopausal women. Hum Reprod 1993; 8: 1487-90.

Cohen J et al. Assisted hatching using micro-manipulation, Human Reproduction 1990; 5: 7-13.

Damario A, Rosenwaks Z. Repeated implantation failure: the preferred therapeutic approach in Assisted Reproductive Techniques Eds, Gardener DK, Weissman A, Howles CM, Shoham Z. Martin Dunitz, London 2001; Chap 45: pp 543-60.

Donnez J, Dolman, Demille, et al. Livebirth after orthotopic transplantation of cryopreserved ovarian Tissue. Lancet 2004; 364: 1405-10.
Fauser BC. Endocrine Profiles after Triggering of Final Oocyte Maturation with GnRH Agonist after Cotreatment with the GnRH Antagonist Ganirelix during Ovarian Hyperstimulation for in Vitro Fertilization. J Clin Endocrinol Metab 2002; 87: 709-15.

Gardener DK, Vella P, Lane M, et al. Culture and transfer of human blastocysts increases implantation rates. Fertil Steril 1998; 69: 84-8.

Gardner DK, Lane M. Embryo culture in Assisted Reproductive Techniques Eds. Gardener DK, Weissman A, Howles CM, Shoham Z. Martin Dunitz, London 2001; Chap 16: pp 204-22.

Grace J, El-Toukhy T, Scriven P, et al. Three hundred and thirty cycles of pre-implantation diagnosis for serious genetic disease: clinical considerations affecting outcome. BJOG 2006; 113: 1393-401.

Jansen R. Dizygotic and monozygotic twinning after IVF. ANZJOG 2006; 46 Suppl 1: S17-19.

Lane M, Gardner DK. Embryo culture medium: which is the best? Clinical Obstetrics and Gynaecology 2007; 21: 85-100.

Lass A. Investigation of the infertile couple for assisted conception in In Vitro Fertilization and Assisted Reproduction. Ed. Brinsden PR. Parthenon Publishing Group, New York 1999; Chap 2: 13-17.

Lavery SA, Winston RML. Clinical experience with PGD at Hammersmith 1989-1998 in Towards Reproductive Certainty. Eds. Jansen R, Mortimer D. Parthenon Publisher Group, U.K. 1999; 49: 397-404.

Mierow J, Levron T, Elder-Geva I, et al. Pregnancy after transplantation of cryopreserved ovarian tissue in a patient with ovarian failure after chemotherapy. N Engl J Med 2005; 353: 318-21.

$\mathrm{Ng}$ EHY, Ho PC. Ageing and ART: a waste of time and money. Clinical Obststrics and Gynaecology. Ed. Norman RJ. Bailliere Tindall, London 2007; 21: 5-20.

Palermo J, Devroey P, Vandervorst J, Nagy M, Van Steirteghem A. Do we treat the male or his gamete? Hum Reprod 1992; 13 (suppl 1): 178-85.

Palermo J, Raffaelli R, Hariprashad JJ, et al. ICSI: technical aspects in Assisted Reproductive Techniques Eds. Gardener DK, Weissman A, Howles CM, Shoham Z. Martin Dunitz, London 2001; 11: 147-57.

Porcu E. Oocyte cryopreservation in Assisted Reproductive Techniques. Eds: Gardner DK, Weissman A, Howles CM, Shoham Z. Martin Dunitz, New York 2001; Chap 18, pp 233-42.

Rothenberg L. Baby M, the surrogate contract and the health Care and professional unanswered questions. Law Med. Health Care 1988; 16: 113-20.

Schatten G. Fertilization in Molecular biology in Reproductive Medicine. Parthenon Publishing Group, London 1999; 14: 305-10.

Schoolcraft WB. Embryo transfer in Assisted Reproductive Techniques Eds. Gardener DK, Weissman A, Howles CM, Shoham Z. Martin Dunitz, London 2001; 50: 623-6.

Scott LA, Smith S. Pronuclear embryo transfers. Hum Reprod 1998; 13: 1003-13. 
Serhal P. Assisted Reproductive technology and older women in Good Clinical Practice in Assisted Reproduction. Eds, Serhal P, Overton C. Cambridge University Press, Cambridge 2004; 19: $319-21$.

Serhal P. Assisted Reproductive technology and older women in Good Clinical Practice in Assisted Reproduction. Eds, Serhal P, Overton C. Cambridge University Press, Cambridge 2004; 19: 319-321.

Suikkari A, Soderstrom-Anttila V. In vitro maturation of eggs: is it really useful? Clinical Obstetrics and Gynaecology 2007; 21-1: 145-55.

Staessen C, Platteau P, Van Assche E, et al. Comparison of blastocyst transfer with or without pre implantation diagnosis for aneuploidy screening in couples with advanced age: a prospective randomised controlled trial. Hum Reprod 2004; 19(12): 2849-58.

Tarlatzis BC, Bili H. Antagonistics of GnRH in Assisted Reproductive Techniques. Eds. Blazer S, Zimmer EZ, Martin Dunitz, London 2001; 40: 493-500.

Tarlatzis BC, Kolibianakis EM. GnRH agonists vs antagonists. Best Pract Res Clin Obstet Gynaecol 2001; 21: 1. 57-65 2007.

Velga A, Boiso I. Assisted hatching in Assisted Reproductive Techniques. Martin Dunitz, London 2001; 12: 164-9.

Wickland ML, Hamberger L. Transvesical and transvaginal approaches for the aspiration of follicles by the use of ultrasound. Ann NY Acad Sci 1985; 442: 683. 